\title{
RETRACTED ARTICLE: Physcion 8-0- $\beta$-glucopyranoside mediates the NLRP3-associated pyroptosis and cell metastasis in the human osteosarcoma cells via ER stress activation
}

\author{
Baogang Tian ${ }^{1} \cdot$ Zhen Hua $^{1} \cdot$ Zhijiong Wang $^{1} \cdot$ Jianwei Wang ${ }^{1}$ \\ Received: 27 August 2019 / Accepted: 30 January 2020 / Published online: 18 February 2020 \\ (C) Springer-Verlag GmbH Germany, part of Springer Nature 2020
}

The Editor in Chief retracted this article (1) because of significant concerns regarding a number of images presented in Figure $6 c$, which question the integrity of the data. It appears a number of the histopathology images have been published previously in unrelated articles $(2,3,4)$. In additional, it appears that an image used in Figure 1c (SAOS-2 at 0uM) has also appeared in unrelated articles with different labels $(5,6)$.

The authors were unable to provide raw data and stated that the figures were obtained through a commercial lab hired to perform some of the experiments for this study.

Author B Tian agrees to this retraction. The other authors have not responded to any correspondence from the editor or publisher about this retraction.

The online version of this article contains the full text of the retracted article as electronic supplementary material.

\section{References}

1) Tian, B., Hua, Z., Wang, Z. et al. Physcion 8-O- $\beta$ glucopyranoside mediates the NLRP3-associated pyroptosis and cell metastasis in the human osteosarcoma cells via ER stress activation. Naunyn-Schmiedeberg's Arch Pharmacol (2020). https://doi.org/10.1007/s00210-020-01836-y.

Electronic supplementary material The online version of this article (https://doi.org/10.1007/s00210-020-01836-y) contains supplementary material, which is available to authorized users.

Jianwei Wang

baogangtwx@163.com

1 Department of Orthopedics, Wuxi Traditional Chinese Medicine Hospital, Nanjing University of Chinese Medicine, No.8 Zhong'nan Road, Wuxi 214071, Jiangsu, China
2) Wang, Q. et al. Physcion 8-O- $\beta$-glucopyranoside inhibits clear-cell renal cell carcinoma bydownregulating hexokinase II and inhibiting glycolysis. Biomedicine \& Pharmacotherapy (2018) https://doi.org/10.1016/j.biopha.2018.05.013.

3) Pan, X., Wang, C., Li, Y., Huang, L. Physcion induces apoptosis through triggering endoplasmic reticulum stress in hepatocellular carcinoma. Biomedicine \& Pharmacotherapy (2018) https://doi.org/10.1016/j.biopha.2018.01.148.

4) Li, Z., Zhang, L., Gao, M. et al. Endoplasmic reticulum stress triggers Xanthoangelol-induced protective autophagy via activation of JNK/c-Jun Axis in hepatocellular carcinoma. J Exp Clin Cancer Res 38, 8 (2019). https://doi. org/10.1186/s13046-018-1012-z.

5) Zhang, J., Wang, Q., Wang, Q. et al. Chrysophanol exhibits anti-cancer activities in lung cancer cell through regulating ROS/ HIF-1a/VEGF signaling pathway. Naunyn-Schmiedeberg's Arch Pharmacol 393, 469-480 (2020). https://doi.org/10.1007/ s00210-019-01746-8.

6) Liu, K., Gao, H., Wang, Q., Wang, L., Zhang, B., Han, Z., Chen, X., Han, M., Gao, M. Hispidulin suppresses cell growth and metastasis by targeting PIM 1 through JAK 2/STAT 3 signaling in colorectal cancer. Cancer Science (2018) https:// doi.org/10.1111/cas.13575. 